\title{
The CME link to geomagnetic storms
}

\author{
Nat Gopalswamy \\ NASA Goddard Space Flight Center, Greenbelt, MD 20771, USA \\ email: nat.gopalswamy@nasa.gov
}

\begin{abstract}
The coronal mass ejection (CME) link to geomagnetic storms stems from the southward component of the interplanetary magnetic field contained in the CME flux ropes and in the sheath between the flux rope and the CME-driven shock. A typical storm-causing CME is characterized by (i) high speed, (ii) large angular width (mostly halos and partial halos), and (iii) solar source location close to the central meridian. For CMEs originating at larger central meridian distances, the storms are mainly caused by the sheath field. Both the magnetic and energy contents of the storm-producing CMEs can be traced to the magnetic structure of active regions and the free energy stored in them.
\end{abstract}

Keywords. Sun: coronal mass ejections (CMEs), solar wind, magnetic fields, interplanetary medium

\section{Introduction}

A geomagnetic storm occurs when the southward magnetic field component $\left(B_{s}\right)$ of an interplanetary (IP) structure reconnects with Earth's magnetic field thereby allowing the entry of the solar wind energy into the magnetosphere. The intensity of the geomagnetic storm is expressed using a number of indices. Here we use the Dst (disturbance storm time) index, which represents the average change in the horizontal component of Earth's magnetic field (in units of nT) brought about by the geomagnetic storm at four lowlatitude stations (see http://swdcwww.kugi.kyoto-u.ac.jp/dstdir/dst2/onDstindex.html). A storm is indicated when Dst $\leqslant-50 \mathrm{nT}$ (Loewe \& Prölss 1997); Dst $\leqslant-100 \mathrm{nT}$ indicates intense storms. Alfven waves in the solar wind, corotating interaction regions (CIRs) and coronal mass ejections (CMEs) are the sources of $B_{s}$ in the IP magnetic field (IMF; Lindsay et al. 1995; Echer, Gonzalez \& Alves 2006; Xu et al. 2009). CMEs cause the most intense of the storms (see e.g., Zhang et al. 2007; Gopalswamy, Yashiro \& Akiyama 2007). The ability of an interplanetary structure in causing a geomagnetic storm is referred to as geoeffectiveness. The geoeffectiveness of IP structures has been well established by the 1990s (e.g., Gosling 1993; Tsurutani et al. 1995). The focus in this paper is on the geoeffectiveness of CMEs observed near the Sun.

It is easy to understand the CME link to geomagnetic storms from the following empirical relationship between the $D s t$ index (nT) and the speed ( $V$ in $\mathrm{km} / \mathrm{s}$ ) and the magnitude of the out-of-the-ecliptic component of the IMF $\left(B_{s}=-B_{z}\right.$ in nT) of the solar wind structure:

$$
\text { Dst }=-0.01 V B_{z}-32 .
$$

This empirical relation was obtained by Gopalswamy et al. (2008) for a subset of interplanetary CMEs (ICMEs) known as magnetic clouds (MCs; Burlaga et al. 1981). For $V=1000 \mathrm{~km} / \mathrm{s}$ and $B_{z}=60 \mathrm{nT}$, one gets $D s t=-632 \mathrm{nT}$, which is roughly the maximum recorded value of the Dst index since solar cycle 19. The Carrington event (1859 September 1) is supposed to have caused a bigger storm, which is not fully understood (see Siscoe, Crooker \& Clauer 2006, and references therein). The dependence 
of Dst on $V$ and $B_{z}$ in Equation 1.1 seems to be firmly established (see e.g., Wu \& Lepping 2002). We shall discuss the applicability of this relationship to non-cloud ejecta in a later section. The $B_{z}$ dependence of $D s t$ is fundamental in that only when $B_{z}$ is negative (southward) that a geomagnetic storm occurs. The speed $V$ is ultimately related to the CME speed at the Sun modified by the solar wind. Statistical studies have shown that the magnetic field strength in ICMEs is weakly correlated with the ICME speed (Gonzalez et al. 1998), which seems to hold even when the CME speed is used in place of the ICME speed (Gopalswamy et al. 2008). Thus the dependence of Dst on $V$ and $B_{z}$ can ultimately be traced to the magnetic properties of the source active regions and the kinematics of CMEs. The CME link can be further understood in terms of the CME source location on the Sun, magnetic properties of the source active regions, the spatial and magnetic structures of CMEs, the CME speed evolution, and the phase of the solar cycle. Towards this end, we use geomagnetic storms from solar cycle 23 because only during this cycle we have continuous CME observations that can be correlated with geomagnetic storms. We also consider only intense storms ( $D s t \leqslant-100 \mathrm{nT}$ ) because these storms can be directly linked to CMEs (Zhang et al. 2007; Gopalswamy, Yashiro \& Akiyama 2007).

\section{CME source connection}

The solar source of an intense geomagnetic storm can be a CME or a CIR. A CME can be traced to a closed magnetic field region on the Sun such as an active region or a quiescent filament region. The CME is often accompanied by a flare in soft X-rays and/or in $\mathrm{H}_{\alpha}$. The flare location on the disk is usually defined as the solar source of the CME. Imaging observations in EUV indicate the flare location, the surrounding dimming region and wave disturbances associated with the CMEs. Microwave imaging can also indicate the location of the filament eruption associated with the CME and the posteruption arcade (flare). Thus the CME source can be identified in a number of ways, ultimately giving the heliographic coordinates of the source location, which is the point of maximum flare brightness in $\mathrm{H}_{\alpha}$, EUV, or X-rays. Details of how one can identify the source regions of ICMEs and geomagnetic storms can be found in Gopalswamy, Yashiro \& Akiyama (2007). In the case of CIRs, it is fairly straightforward to identify the underlying coronal hole as the solar source. The central meridian passage of the coronal hole occurs a couple of days before the arrival of the high speed stream at Earth. In the in-situ data, CIRs appear as regions of enhanced density and temperature during the transition from slow to high speed solar wind.

Figure 1 shows the heliographic coordinates of CMEs that produced large geomagnetic storms $(D s t \leqslant-100 \mathrm{nT})$ during solar cycle 23 . The sizes of the circles denote the intensity of the storms: largest circles correspond to super storms (Dst $\leqslant-300 \mathrm{nT})$. The medium circles represent storms with $D s t \leqslant-200 \mathrm{nT}$ but $>-300 \mathrm{nT}$ (secondlevel storms). The smallest circles correspond to storms with $D s t>-200 \mathrm{nT}$ (first-level storms). Note that the superstorms are located roughly within a central meridian distance (CMD) of $\sim 15^{\circ}$ (inner region) with equal number of events in the eastern and western hemispheres. There are only 4 second-level storms in the eastern hemisphere, but 7 in the western hemisphere. Furthermore, three of the western second-level storms have their solar sources beyond the inner region. The first-level storms show western bias both in number and longitudinal spread of source locations. There is only one source beyond a CMD of $75^{\circ}$ in each hemisphere. Thus, we see that CMEs originating closer to the disk center have a better opportunity to produce intense magnetic storms (see also Wang et al. 2002; Zhang et al. 2007; Gopalswamy, Yashiro \& Akiyama 2007). The slight western bias 

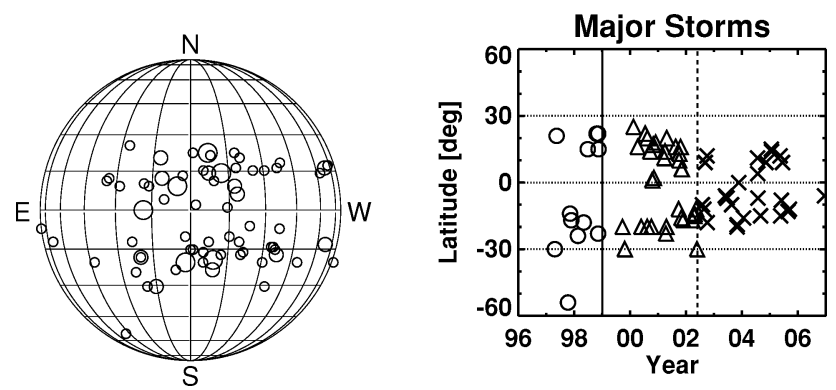

Figure 1. (left) Solar source locations of large geomagnetic storms of solar cycle 23 distinguished according to the storm strength. (right) the source latitudes as a function of time with the three phases of the solar cycle shown separately (circles: rise phase-1996 to 1998; triangles: maximum phase -1999 to 2002.6; crosses: declining phase - 2002.6 to 2007).

may be due the fact that CMEs undergo a slight eastward deflection because of the solar rotation as was pointed out by Gosling et al. (1987a). Since CMEs are ejected radially, one expects direct impact on the magnetosphere when they originate from close to the disk center.

The latitudes of CME sources plotted as a function of time in Fig. 1 show that the storm-producing CMEs typically originate within $\pm 30^{\circ}$ latitude (there is only one exception in the beginning of the solar cycle). During the solar minimum, the sources are at slightly higher latitudes compared to the maximum and declining phases. The latitude distribution of the solar sources resembles the sunspot butterfly diagram, suggesting that most of the storm-producing CMEs originate from active regions. Solar rotation brings active regions closer to the disk center; solar activity brings active regions closer to the disk center over a longer time scale. During solar minimum, the global dipolar field also channels CMEs closer to the equator (even though the active regions themselves are located at higher latitudes).

\section{The kinematic connection}

The kinematic link to the geomagnetic storms (the V part in Equation 1.1) can be understood from Fig. 2, which shows the speed and width distributions of CMEs that produced major geomagnetic storms (Dst $\leqslant-100 \mathrm{nT})$ during cycle 23 . The speed distribution peaks in the $700 \mathrm{~km} / \mathrm{s}$ bin and has an average speed of $999 \mathrm{~km} / \mathrm{s}$. The width $(W)$ distribution is dominated by the single bin at $360^{\circ}$ (halo CMEs). In fact $68 \%$ of the CMEs are full halos $\left(W=360^{\circ}\right)$, which increases to $92 \%$ when partial halos are included (i.e., all CMEs with $W \geqslant 120^{\circ}$ ). Even the small number of non-halos are wide (average width $\sim 88^{\circ} .7$ ). No CMEs can be found in the bins below the $50^{\circ}$ bin. The fraction of halo CMEs in a population is indicative of the fact that these CMEs are very energetic compared to the general population of CMEs. This can be easily seen from the observation that the average speed of halo CMEs is $\sim 1000 \mathrm{~km} / \mathrm{s}$ (Gopalswamy 2004). Furthermore, faster CMEs are known to be wider (Gopalswamy et al. 2001a, 2009a), implying that halo CMEs must be inherently wider and hence more energetic. Faster CMEs also decelerate more within the coronagraphic field of view (Gopalswamy et al. 2001a). Accordingly, there is a clear bias towards negative acceleration in Fig. 2. The average acceleration is 

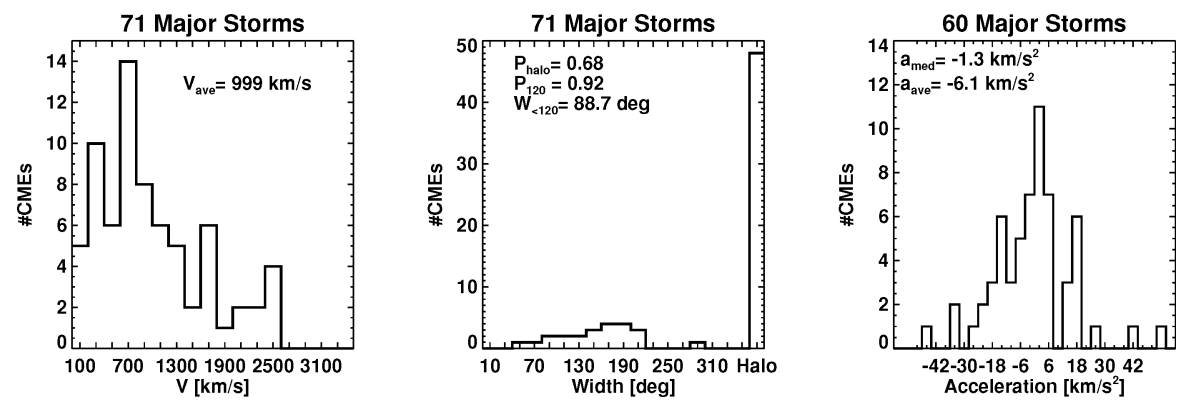

Figure 2. Distributions of speed, width and acceleration of CMEs associated with major geomagnetic storms during cycle 23. The average speed of CMEs is nearly twice that of the general population. Fraction of full halo CMEs in the population $\left(\mathrm{P}_{\text {halo }}\right)$, Fraction of wide CMEs $\left(\mathrm{P}_{120}\right)$, and non-halo CMEs $\left(\mathrm{W}_{<120}\right)$ are shown on the width plot. Note that the halo CME bin is very tall compared to other bins in the width distribution. The acceleration distribution is clearly skewed toward negative values, consistent with the high speed of the CMEs.

$-6.1 \mathrm{~ms}^{-2}$ and the median value is $-1.3 \mathrm{~ms}^{-2}$. These values are approximate because of projection effects (the height-time measurements used for determining the speed and acceleration are made in the sky plane).

CMEs launched into the interplanetary medium are subject to a drag force that depends on the CME speed and the ambient physical conditions (speed, density and drag coefficient). The drag force tends to slow down fast CMEs and speed up slow CMEs, which can be represented by an interplanetary acceleration, $a=-0.0054\left(V_{C M E}-406\right)$, where $a$ is the acceleration in $\mathrm{ms}^{-2}$ and $V_{C M E}$ is the CME speed $(\mathrm{km} / \mathrm{s})$ measured near the Sun (Gopalswamy et al. 2001b). The number $406 \mathrm{~km} / \mathrm{s}$ corresponds to the average speed of the slow solar wind into which the CMEs are launched. For speeds higher than $\sim 1000 \mathrm{~km} / \mathrm{s}$, the acceleration is a quadratic function of the initial speed (Gopalswamy 2002). The interplanetary acceleration ranges from $\sim-10 \mathrm{~ms}^{-2}$ for fast CMEs to $\sim 2$ $\mathrm{ms}^{-2}$ for slow CMEs. Figure 3 shows the relationship between the speeds measured at the Sun and at $1 \mathrm{AU}$ for a large number (184) of events during cycle 23. The plot includes both types of ICMEs: those with flux rope structure (MCs) and without (non-MCs). The CME speeds at the Sun are in the range $\sim 100$ to $>3000 \mathrm{~km} / \mathrm{s}$, but the corresponding speeds at Earth are in the range $\sim 300$ to $\sim 1500 \mathrm{~km} / \mathrm{s}$. The narrower range of speeds at Earth is a direct result of the interaction between CMEs and the solar wind. The speed at $1 \mathrm{AU}(V)$ depends on $V_{C M E}$ according to $V=365+0.16 V_{C M E}+1.4 \times 10^{-5} V_{C M E}^{2}$. When $V_{C M E} \sim 400 \mathrm{~km} / \mathrm{s}$, we see that $V \sim V_{C M E}$ because these CMEs are not affected by the solar wind drag. Lindsay et al. (1999) had obtained a linear relationship between $\mathrm{V}$ and $\mathrm{V}_{C M E}$ for a smaller range of speeds.

The ICME speed in Fig. 3 is slightly higher for MCs compared to non-MCs (see Fig.3). This is likely to be due to the fact that the observing spacecraft passes through the nose of the ICMEs when they are MCs and hence record the maximum speed. For non-MCs, the spacecraft may pass through the flanks of the CMEs at varying distances from the nose, so a lower speed is recorded and also varies significantly from event to event. These effects can be directly linked to the CME source location on the Sun: MCs generally result from CMEs originating close to the disk center, while non-MCs result from CMEs originating at larger central meridian distance (Gopalswamy 2006b). $\mathrm{V}_{C M E}$ is also subject to projection effects, which are more pronounced for MCs than non-MCs, again due to their different locations with respect to the Sun center. 


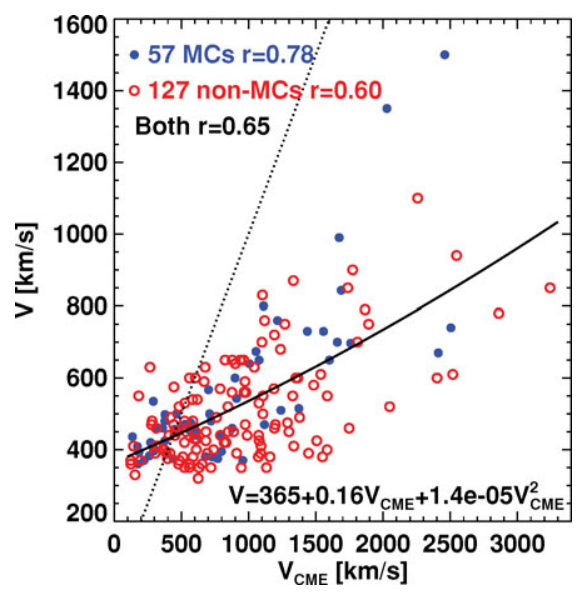

Figure 3. The relationship between the speeds measured at the Sun and at $1 \mathrm{AU}$ for a large number of CMEs during cycle 23. Fifty seven CMEs that resulted in magnetic clouds (MCs, filled circles) and 127 that resulted in non-clouds ICMEs (non-MCs, open circles) are distinguished. The bisector $\left(V=V_{C M E}\right)$ and the quadratic fit to the data points are shown.

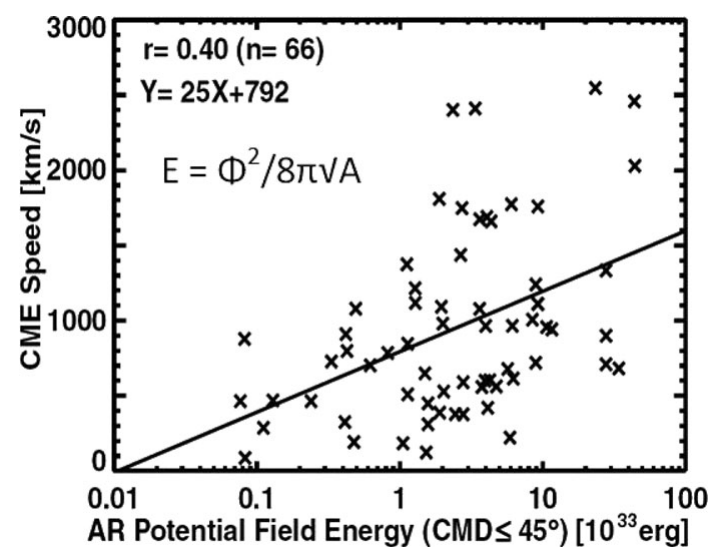

Figure 4. Scatterplot between the active region (AR) potential energy and the CME speed for 66 events observed during cycle 23.

\section{The magnetic connection}

\subsection{CME speed and active region free energy}

The relation between CME speed and the magnetic potential energy of the source active regions is shown in Fig. 4. The plot includes only those CMEs that resulted in large geomagnetic storms (same as in Fig. 1) and MCs at 1 AU. It is believed that the free energy in an active region is of the order of the magnetic potential energy (MacKay et al. 1997). This gives a useful way of estimating the maximum free energy that can be stored in an active region. Fastest CMEs originate from active regions of highest magnetic potential energy, even though the correlation between the two quantities is only moderate (correlation coefficient $r=0.40$ ). From Fig. 4 we see that the maximum active region potential energy during cycle 23 did not exceed $\sim 4 \times 10^{34} \mathrm{erg}$, suggesting that the maximum free energy that can be stored cannot be more than this value. If all the free energy were to be released in a single CME, the CME kinetic energy could be as high as $\sim 4 \times 10^{34} \mathrm{erg}$. In reality, the maximum CME kinetic energy observed during cycle 23 was $\sim 1.2 \times 10^{33} \mathrm{erg}$, estimated for the CME on 2003 October 28 (Gopalswamy et al. 
230 shocks in 1996-2006

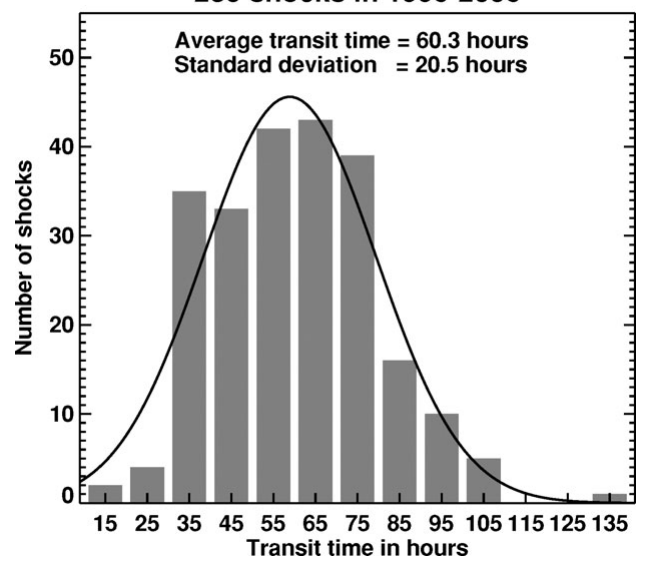

Figure 5. Transit time distribution of CME-driven shocks detected at Sun-Earth L1 during solar cycle 23. A Gaussian fit to the histogram is overlaid on the distribution. The average of the distribution and the standard deviation are noted on the plot.

2005). This means that all the free energy stored in an active region need not be released in a single CME; several CMEs may occur over a period of time to exhaust all the free energy. The CME speed distribution indicates a sharp cutoff beyond $\sim 3500 \mathrm{~km} / \mathrm{s}$ (see e.g., Gopalswamy 2006a). Assuming that the maximum speed attained by CMEs is $\sim 4000$ $\mathrm{km} / \mathrm{s}$ and using the maximum CME mass of $\sim 10^{17} \mathrm{~g}$, one can estimate the maximum kinetic energy to be $\sim 8 \times 10^{33} \mathrm{erg}$. This is again consistent with the observations of magnetic potential energy during cycle 23 .

The maximum energy suggested in Fig. 4 has some interesting implications. The active region energy $E$ can be written as $E=\Phi^{2} / 8 \pi \sqrt{A}$, where $\Phi$ and $A$ are the active region magnetic flux and area, respectively. The maximum energy implies a limit to the maximum field strength $(B)$ and the area of the active regions. The field strengths in sunspots has been estimated to have a maximum value of $\sim 6000 \mathrm{G}$ (Livingston et al. 2006). Active region areas as high as 5000 millionths of solar hemisphere (msh) have been reported in the literature (Newton 1955). Combining these two, one can estimate the limit of active region energy as $E \sim 2.7 \times 10^{36} \mathrm{erg}$. This is a factor of $\sim 70$ higher than the observed value during cycle 23 . The maximum active region area during cycle 23 was $2610 \mathrm{msh}$ in AR 10486 (Gopalswamy et al. 2005), which is only about half the maximum value reported in Newton (1955). The peak field strength in AR 10486 was $\sim 1700 \mathrm{G}$ (Chumak, Zhang \& Guo 2008). Therefore, the maximum active region potential energy is accordingly smaller $\left(\sim 8 . \times 10^{34} \mathrm{erg}\right.$. $)$

\subsection{CME structure and geomagnetic storms}

CMEs causing geomagnetic storms are generally fast and wide CMEs (see Fig. 2), which means most of them must be shock-driving. The shock surrounds the CME as a bow wave and stands at a distance that depends on the shock strength and the CME size. The average standoff distance corresponds to $\sim 0.5$ day between the shock and ICME arrivals at Earth (Gosling et al. 1987b; Gopalswamy et al. 2008). This means the first thing from a CME that strikes the magnetosphere is the shock. The shock compresses the magnetosphere, which produces a spiky increase in Dst, known as the storm sudden commencement (SSC) because a geomagnetic storm usually follows (Curto, Araki \& Alberca 2007, and references therein). Shocks take anywhere from $\sim 19$ hours to more than four days to reach Earth, as can be seen in Fig. 5, which shows the distribution 


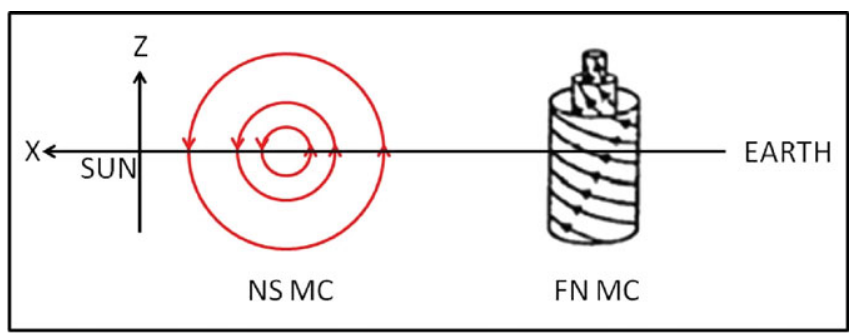

Figure 6. Out-of-the-ecliptic component $\left(B_{z}\right)$ of the interplanetary magnetic field due to NS and FN MCs. For NS MCs, the front has $B_{z}>0$ (not geoeffective) and the back has $B_{z}<0$ (geoeffective). The FN MCs have $B_{z}>0$ throughout the MC interval. For the SN and FS MCs, the arrows point in the opposite direction. The Y-axis points into the plane of the page.

of the transit time of 230 shocks detected during solar cycle 23 . The distribution is roughly symmetric with an average transit time of $60.3 \mathrm{~h}$ (standard deviation of $20.5 \mathrm{~h}$ ). Whether a geomagnetic storm would follow the shock depends critically on the existence of $B_{s}$ somewhere within the interval of interest (IOI), which starts from the shock and ends at the end of the driving ICME. Favorable conditions can occur within the IOI when the sheath and/or the ICME portions contain $B_{s}$. While it is straightforward to identify the shocks in the solar wind data, one has to infer from type II radio burst data (Gopalswamy 2006c) or from coronagraphic observations (Gopalswamy et al. 2009b; Ontiveros \& Vourlidas 2009). Unfortunately, the remote-sensing and in-situ instruments do not detect the same section of the CME-driven shocks.

When the ICME is an MC (flux rope), it is easy to guess the time structure of the Dst index. MCs can be classified into four groups: south-north (SN), north-south (NS), fully-south (FS) and fully-north (FN), depending on the direction of $B_{z}$ in the MC (see Fig. 6). The flux-rope axis of the SN and NS clouds are in the ecliptic plane, so $B_{z}$ is the azimuthal component; it is perpendicular to the ecliptic for FS and FN clouds, so $B_{z}$ is the axial component. The SN and NS MCs have $B_{s}$ in the front and back of the cloud, respectively and hence making them geoeffective. No storm follows FN clouds due to the lack of $B_{s}$. On the other hand, FS MCs are always geoeffective because of the axial $B_{s}$. In addition, if the sheath contains $B_{s}$, then one gets a complex Dst profile depending on the distribution of $B_{s}$ in the IOI. Gopalswamy (2008) reported that the peak of sheath storms precede the MC arrival by $\sim 3.2 \mathrm{~h}$, while the cloud storms peak after $\sim 10.6 \mathrm{~h}$. The delay of course depends on the MC type: the largest is for NS clouds $(18.6 \mathrm{~h})$, the shortest for SN MCs $(5.5 \mathrm{~h})$ and intermediate (9.3h) for FS MCs.

White-light CMEs associated with the MCs typically erupt from very close to the disk center $\left(\mathrm{CMD}<30^{\circ}\right)$. This property is shared by storm-producing CMEs shown in Fig. 1 . On the other hand, CMEs associated with non-MCs originate at larger CMDs on the average. In the extreme case, one observes shocks at $1 \mathrm{AU}$ without a discernible driver when energetic CMEs originate from close to the solar limb. In these cases, only the shock flank is expected to impact Earth, occasionally producing a geomagnetic storm. One of the implications of this geometrical effect is that all ICMEs may contain flux ropes (MCs) but may or may not be observed as flux ropes: the observing spacecraft passes through the nose of the ICME only for MCs; for non-MCs, the observing spacecraft passes through the edges of the ICMEs; for shock flanks, the spacecraft completely misses the ICME part (Marubashi 1997; Gopalswamy 2006a; Riley et al. 2006). The flux rope is thought to be formed due to reconnection in the closed field regions where CMEs originate. The post eruption arcade is supposed to be an indication that a flux rope has formed due to reconnection and erupted (Qiu et al. 2007). It is also possible that the flux rope grew 


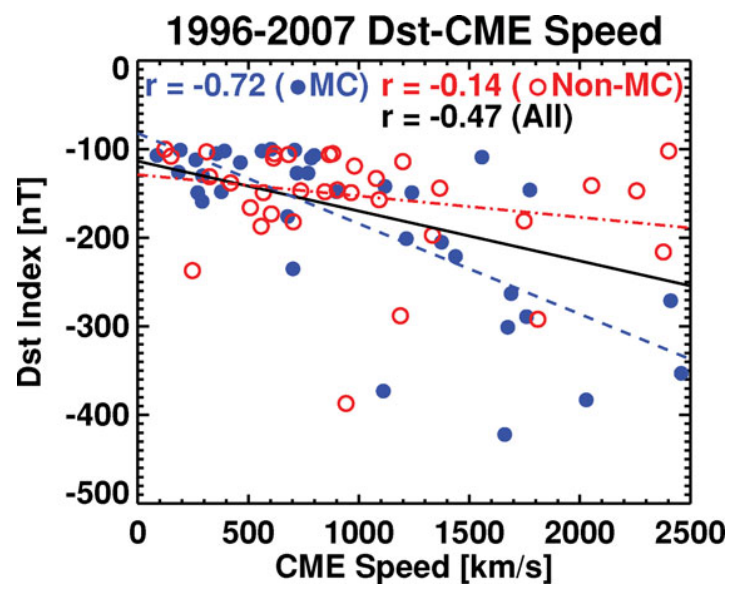

Figure 7. Scatterplot between Dst index and the CME speed for large geomagnetic storms during cycle 23. The storms caused by MCs and non-MCs are distinguished. The correlation coefficients for all storms, MC storms and non-MC storms are shown on the plot.

out of a preexisting flux rope by adding flux during the reconnection process. The flux rope derives its kinetic energy from the free energy stored in the active region. The flux rope axis is typically parallel to the neutral line (or filament) in the active region, while the handedness of the flux rope can be inferred from the sign of the active region helicity (Marubashi 1997). Thus one can even infer the $B_{z}$ part of Equation 1.1, but this is a field of active research.

The glancing impact expected from non-MCs may have consequences for their geoeffectiveness. Figure 7 shows the dependence of $D s t$ on the CME speed for major storms of cycle 23. The overall correlation is modest $(r=-0.47)$, but the CMEs that resulted in MCs show a much better correlation $(r=0.72)$. On the other hand, CMEs resulting in non-MCs show poor correlation $(r=-0.14)$. This can once again be attributed to the fact that non-MCs may strike Earth at different glancing angles. The good correlation between CME speed and Dst index for MCs is somewhat reduced $(r=-0.46)$ when starting from all MCs and compiling the strength of the resulting storms without any restriction on the Dst values (Gopalswamy et al. 2008).

\section{Summary and Discussion}

The primary CME link to the geomagnetic storms arises from the fact that they can introduce an out-of-the ecliptic component to the interplanetary magnetic field that arrives at Earth. Whenever the out-of-the-ecliptic component of the magnetic field in a solar wind structure points to the south, a geomagnetic storm ensues due to a series of physical processes elucidated by Dungey (1961). Figure 8 summarizes the CME connection to geomagnetic storms via Equation 1.1: both the CME speed and the southward magnetic field it contains determine the strength of the storm. The southward component of the magnetic field in a CME arises from their flux-rope structure and in the sheath region due to field line draping around the flux rope and compression. The flux rope originates from the source active region, whereas the sheath field is from the heliosphere. The CME speed depends on the active region free energy, modified by the interaction with the solar wind. Thus both the speed and magnetic field parameters are linked to the free energy and magnetic properties of the source active region. For example, a CME 


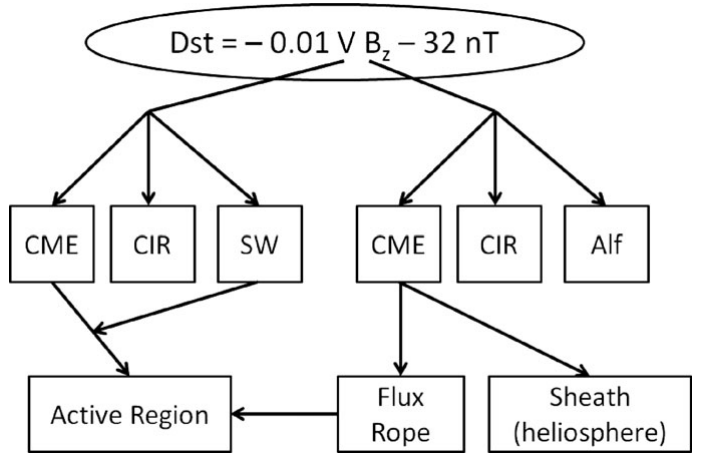

Figure 8. Schematic representation of the CME connection to geomagnetic storms. The empirical relation connecting the Dst index to the speed $(V)$ and southward magnetic field component $\left(B_{s}\right)$ of the interplanetary structures is from Gopalswamy et al. (2008). Other sources of $V$ and $B_{z}$ such as the solar wind (SW), Alfven waves (Alf), CIR, and shock sheath are also shown.

that results in an MC with larger magnetic flux and/or helicity is launched from the Sun with a higher initial speed (Sung et al. 2009).

Since CMEs are the main source of major geomagnetic storms, one would like to predict the CME speed and the magnitude of $B_{s}$ from the observed properties of the active regions (Falconer et al. 2009). There have been several attempts to qualitatively link the ICME properties to the active region properties: sign of helicity (Bothmer \& Schwenn 1994; Martin \& McAllister 1997; Yurchyshyn et al. 2001), azimuthal magnetic flux of MCs and the magnetic flux in flare ribbons (Qiu et al. 2007), and axial magnetic flux to the flux in coronal dimming regions (Webb et al. 2000). A detailed review can be found in Démoulin (2008). Predicting the kinematic and magnetic properties of CMEs near the Sun and in the IP medium based on the active region properties is required to provide advanced warning of potential geomagnetic storms (see e.g., Srivastava 2006).

Finally, we note that the importance of $B_{s}$ in causing geomagnetic storms is due to the fact that Earth's magnetic field currently points to the north. Paleomagnetic records and numerical experiments indicate that the polarity of the geomagnetic field can be reversed on geological time scales (Roberts \& Glatzmaier 2000). When Earth's magnetic field points to the south, one has to worry about the north-pointing interplanetary magnetic structures in causing the geomagnetic storms. Accordingly, the north-south magnetic clouds would cause prompt storms rather than the south-north clouds.

\section{Acknowledgements}

The author thanks P. Mäkelä for help with formating and several figures. Work supported by NASA's LWS TR\&T program.

\section{References}

Bothmer, V. \& Schwenn, R. 1994, Space Sci. Revs 70, 215

Burlaga, L., Sittler, E., Mariani, F., \& Schwenn, R. 1981, J. Geophys. Res. 86, 6673

Chumak, O. V., Zhang, H.-Q., \& Guo, J. 2008, Astronomy Reports, 52(10), 852

Curto, J. J., Araki, T., \& Alberca, L. F. 2007, Earth Planets Space 59, p. i

Démoulin, P. 2008, Ann. Geophys. 26, 3113

Dungey, J. W. 1961, Phys. Rev. Lett. 6, 47

Echer, E., Gonzalez, W. D., \& Alves, M. V. 2006, Space Weather 4, S06001

Falconer, D., Moore, R.L., Gary, G. A., \& Adams, M. 2009, ApJ 700, L166

Gonzalez, W. D., Gonzalez, A. L. C., Dal Lago, A. et al. 1998, Geophys. Res. Lett. 25(7), 963 
Gopalswamy, N. 2002, COSPAR Colloquia Series 14, 157

Gopalswamy, N. 2004, in: G. Poletto \& S. T. Suess (eds.), The Sun and the Heliosphere as an Integrated System, (Boston: Kluwer), p. 201

Gopalswamy, N. 2006a, JA\&A 27, 243

Gopalswamy, N. 2006b, Space Sci. Revs 124, 145, doi:10.1007/s11214-006-9102-1

Gopalswamy, N. 2006c, in: N. Gopalswamy, R. A. Mewaldt, \& J. Torsti (eds.), Solar Eruptions and Energetic Particles, Washington, DC: AGU, p. 207

Gopalswamy, N. 2008, J. Atmos. Sol.-Terr. Phy., doi:10.1016/j.jastp.2008.06.010

Gopalswamy, N., Yashiro, S., Kaiser, M. L., Howard, R. A., \& Bougeret, J.-L. 2001a, J. Geophys. Res. 106, 29219

Gopalswamy, N., Lara, A., Yashiro, S., Kaiser, M. L., \& Howard, R. A. 2001b, J. Geophys. Res. 106,29207

Gopalswamy, N., Yashiro, S., Liu, Y., Michalek, G., Vourlidas, A., Kaiser, M. L., \& Howard, R. A. 2005, J. Geophys. Res. 110, A09S15, doi:10.1029/2004JA010958

Gopalswamy, N., Yashiro, S., \& Akiyama, S., 2007, J. Geophys. Res. 112, A06112, doi: 10.1029/2006JA012149

Gopalswamy, N., Akiyama, S., Yashiro, S., Michalek, G., \& Lepping, R. P. 2008, J. Atmos. Sol.-Terr. Phy. 70, 245

Gopalswamy, N., Dal Lago, A., Yashiro, S., \& Akiyama, S. 2009a, Central European Astrophysical Bulletin 33, 115

Gopalswamy, N., Thompson, W. T., Davila, J. et al. 2009b, Solar Phys. 259, 227

Gosling, J. T. 1993, J. Geophys. Res. 98, 18937

Gosling, J. T., Thomsen, M. F., Bame, S. J., \& Zwickl, R. D. 1987a, J. Geophys. Res. 92, 12399

Gosling, J. T., Baker, D. N., Bame, S. J., Feldman, W. C., Zwickl, R. D. \& Smith, E. J. 1987b, J. Geophys. Res. 92, 8519

Lindsay, G. M., Russell, C. T., \& Luhmann, J. G. 1995, J. Geophys. Res. 100, 16999

Lindsay, G. M., Luhmann, J. G., Russell, C. T., \& Gosling, J. T. 1999, J. Geophys. Res. 104, 12515

Livingston, W., Harvey, J. W., Malanushenko, O. V., \& Webster, L. 2006, Solar Phys. 239, 41

Loewe, C. A. \& Prölss, G. W. 1997, J. Geophys. Res. 102, 14209

Martin, S. F. \& McAllister, A. H. 1997, in: N. Crooker, J. A. Joselyn, \& J. Feynman (eds.), Coronal Mass Ejections, Geophys. Monogr. Ser. 99 (Washington, D.C.: AGU), p. 127

Marubashi, K. 1997, in: N. Crooker, J. A. Joselyn, \& J. Feynman (eds.), Coronal Mass Ejections, Geophys. Monogr. Ser. 99 (Washington, D.C.: AGU), p. 147

MacKay, D. H., Gaizauskas, V., Rickard, G. J., \& Priest, E. R. 1997, ApJ 486, 534

Newton, H. W. 1955, Vistas in Astronomy 1(1), 666

Ontiveros, V. \& Vourlidas, A. 2009, ApJ 693, 267

Qiu, J., Hu, Q., Howard, T. A., \& Yurchyshyn, V. B. 2007, ApJ 659, 758

Riley, P., Schatzman, C., Cane, H. V., Richardson, I. G., \& Gopalswamy, N. 2006, ApJ 647, 648

Roberts, P. H. \& Glatzmaier, G. A. 2000, Rev. Mod. Phys. 72, 1081

Siscoe, G., Crooker, N. U., \& Clauer, C. R. 2006, Adv. Sp. Res. 38(2), 173

Srivastava, N. 2006, JAEA 27, 237

Sung, S.-K., Marubashi, K., Cho, K.-S. et al. 2009, ApJ 699, 298

Tsurutani, B. T., Gonzalez, W. D., Gonzalez, A. L. C. et al. 1995, J. Geophys. Res. 100, 21717

Wang, Y. M., Ye, P.Z., Wang, S., Zhou, G. P., \& Wang, J. X. 2002, J. Geophys. Res. 107, 1340

Webb, D. F., Lepping, R. P., Burlaga, L. F., DeForest, C. E., Larson, D. E., Martin, S. F., Plunkett, S. P., \& Rust, D. M. 2000, J. Geophys. Res. 105, 27251

Wu, C. C. \& Lepping, R. P. 2002, J. Geophys. Res. 107, 1346

Xu, D., Chen, T., Zhang, X. X., \& Liu, Z. 2009, J. Atmos. Sol.-Terr. Phy. 57, 1500

Yurchyshyn, V. B., Wang, H., Goode, P. R., \& Deng, Y. 2001, ApJ, 563, 381

Zhang, J. et al. 2007, J. Geophys. Res. 112, A10102, doi:10.1029/2007JA012321 